\title{
Lack of MMP10 exacerbates experimental colitis and promotes development of inflammation-associated colonic dysplasia
}

\author{
Felicitas L Koller ${ }^{1}$, E Ashley Dozier ${ }^{2}$, Ki Taek Nam ${ }^{1,3,4}$, Mei Swee ${ }^{5}$, Timothy P Birkland ${ }^{5}$, William C Parks ${ }^{5}$ and \\ Barbara Fingleton ${ }^{2}$
}

Inflammatory bowel diseases (IBD) such as ulcerative colitis (UC) represent serious health burdens because of both the tissue-damaging disease itself and an elevated risk of colon cancer. The increased expression of many members of the matrix metalloproteinase (MMP) family of enzymes that occurs in colitis has long been associated with the destructive nature of the disease. Recent findings in cancer and other MMP-associated diseases, however, led us to question whether MMPs are indeed detrimental in the setting of colitis. Here, we focus on a single MMP family member, MMP10, and assess its role in a murine model of colonic tissue damage induced by dextran sulfate sodium (DSS) treatment. Using mice genetically deficient for MMP10, we find that absence of this enzyme leads to significantly worse disease scores and failure to resolve inflammation even after extended recovery periods. We show that MMP10 is produced predominantly by infiltrating myeloid cells in both murine and human colitis. Through bone marrow transplant experiments, we confirm that bone marrow-derived MMP10 contributes to colitis severity. Mice lacking MMP10 have a significantly higher propensity for development of dysplastic lesions in the colon after two rounds of DSS exposure. Thus, we conclude that MMP10 is required for resolution of DSS-induced colonic damage, and in its absence, chronic inflammation and ultimately dysplasia occurs.

Laboratory Investigation (2012) 92, 1749-1759; doi:10.1038/labinvest.2012.141; published online 8 October 2012

KEYWORDS: bone marrow-derived cells; cancer; colitis; protease; resolution

Inflammatory bowel disease (IBD) affects $\sim 2$ million people in the United States alone. Ulcerative colitis (UC), one of the most common manifestations of IBD, is characterized by chronic relapsing inflammation of the large bowel that spreads from the rectum toward the colon-ileal junction. Clinical symptoms include diarrhea, rectal bleeding, passage of mucus, urgency, and abdominal pain. ${ }^{1}$ UC produces significant morbidity in affected individuals through the burden of its clinical symptoms and by increasing patients' risk of colorectal cancer. ${ }^{2}$ Histologically, UC is characterized by inflammatory cell infiltration, epithelial cell destruction, and continuous mucosal inflammation and ulceration that spread proximally from the rectum. Currently, UC is without a cure and treatment involves the administration of disease-modifying agents that often carry substantial side effects. $^{3}$ Surgical treatment is also not without significant morbidity, including infection, infertility, sexual dysfunction, and fecal incontinence. Ideally, a treatment of UC would induce and maintain remission, promote mucosal healing, avoid surgical intervention, and reduce cancer risk. ${ }^{4}$

Matrix metalloproteinases (MMPs) initially presented an attractive target for IBD treatment because ulcer biopsies from patients with IBD had high levels of several enzymes including MMPs $1,3,7,9,10$, and $12 .^{5-9}$ In humans, MMPs are a family of 24 proteases with unique zinc-containing catalytic domains. Although there were several favorable reports on the use of synthetic MMP inhibitors in murine colitic damage induced with dextran sodium sulfate (DSS) or trinitrobenzensulfonic acid (TNBS), ${ }^{10,11}$ the clinical use of MMP inhibitors fell into disfavor when human cancer trials using MMP inhibitors had negative results. ${ }^{12}$

\footnotetext{
${ }^{1}$ Department of Surgery, Vanderbilt University School of Medicine, Nashville, TN, USA; ${ }^{2}$ Department of Cancer Biology, Vanderbilt University School of Medicine, Nashville, TN, USA; ${ }^{3}$ Nashville Department of Veterans Affairs Medical Center, Vanderbilt University School of Medicine, Nashville, TN, USA; ${ }^{4}$ Epithelial Biology Center, Vanderbilt University School of Medicine, Nashville, TN, USA and ${ }^{5}$ Center for Lung Biology, University of Washington, Seattle, WA, USA Correspondence: Dr B Fingleton, PhD, Department of Cancer Biology, Vanderbilt University, 771 PRB, 2220 Pierce Avenue, Nashville, TN 37232-6840, USA. E-mail: Barbara.fingleton@vanderbilt.edu 
The roles of MMPs in human disease were reappraised following the disappointing cancer trials. It became clear that MMPs had more diverse, complex functions than previously believed. In addition to matrix proteins, chemokines, cytokines, receptors, and antimicrobial peptides are now recognized as in vivo substrates for MMPs. ${ }^{13,14}$ The connection between MMP expression and IBD is well established, but how MMP function may be detrimental or beneficial in the course of the disease has remained unclear. A large part of the complexity stems from the myriad roles that MMPs may play in vivo.

Here we focus on one particular MMP, MMP10, also known as stromelysin-2. MMP10 is a rational target for investigation in IBD because its expression has been described at healing ulcer edges in human specimens of UC. ${ }^{8}$ As is the case for many MMPs, the timing of MMP10 expression associates it with disease pathophysiology. However, the reported localization of human MMP10 to healing edges suggests a possible role in disease resolution rather than disease progression. Thus, we used $M m p 10^{-/-}$mice to ask whether MMP10 plays a beneficial or detrimental role in colonic injury using the DSS model. Furthermore, we investigate the consequences of MMP10 ablation on the most serious longterm outcome of chronic colonic injury, that is, neoplasia.

\section{MATERIALS AND METHODS}

Mice

Breeding pairs of $\mathrm{Mmp} 10^{-1-}$ mice, on the C57BL/6 background, were transferred from the University of Washington and used to establish colonies in both a specific pathogen-free room (after re-derivation at Jackson Labs, Bar Harbor, ME, USA) and regular mouse housing. Similarly, C57BL/6j control mice were purchased from Jackson Labs and used to establish breeding colonies with housing in cages directly adjacent to the $M m p 10^{-1-}$ colony. For all colitis experiments, 8-week-old male mice were used, as female mice showed greater variation in the response to DSS (as has been reported ${ }^{15}$ ). Before initiation of our studies, all animal experiments were approved by the local institutional animal care and use committee.

\section{Experimental Colitis Model}

A $2.5 \%$ aqueous solution of DSS (MW $=36000-50000$; MP Biochemicals, Solon, OH, USA) was provided ad libitum to the mice in water bottles for 7 days. The amount of DSS solution in the bottles was recorded daily to ensure similar exposures for all cages. After 7 days, cohorts of mice were immediately killed (7 day), returned to regular water for 3 days and then killed (10 day), returned to regular water for 7 days and then killed (14 day), or returned to regular water for 14 days before being killed (21 day). All mice were weighed daily from the first day of DSS exposure to the day of killing.

\section{Chronic Colitis}

To induce a chronic colitis, mice were put through 2 separate 4 -day exposures to $3 \%$ DSS that were 16 days apart. The mice were maintained for a further 4 weeks before killing.

\section{Bone Marrow Transplants}

All bone marrow transplant experiments were performed with mice in the specific pathogen-free barrier facility. Bone marrow was harvested from the femurs of 4-week-old donor female mice, either control C57BL/6 or $\mathrm{Mmp10}^{-/-}$, and rinsed twice in sterile PBS. The bone marrow cells were counted and diluted with sterile PBS to a concentration of $2 \times 10^{7}$ cells per $\mathrm{ml}$. The 5 -week-old recipient male mice were lethally irradiated using a split dose regimen with two doses of $4 \mathrm{~Gy}$ given $4 \mathrm{~h}$ apart, using an RS2000 X-ray irradiator (RadSource, Suwanee, GA, USA). At $3 \mathrm{~h}$ after the second radiation dose, the mice received $2 \times 10^{6}$ donor bone marrow cells injected intravenously via the tail vain. Recipient mice were weighed daily for the first week following transplant and weekly thereafter until 4 weeks after transplant. They were then administered 2.5\% DSS for 7 days as described previously, switched to regular water for a 3-day recovery period, and then killed.

\section{Histology and Scoring}

Colons were removed from the killed mice, rinsed with PBS, and then cut open longitudinally before being rolled into 'Swiss rolls'. The Swiss rolls were held together with a 30 -gauge needle, fixed in $10 \%$ formalin and embedded in paraffin. Sections $(5 \mu \mathrm{m})$ of the paraffin-embedded tissue were cut and stained for basic histology using hematoxylin and eosin (H\&E) and periodic acid-Schiff (PAS) stains, all purchased from Sigma (St Louis, MO, USA).

A colitis damage score was generated from digital images of PAS-stained sections, by first measuring the distance around the intact muscularis using the AVP Universal Desktop Ruler software. The areas of ulceration were then measured and given the following scores: $1=1 / 3$ of the bottom of the crypt injured; $2=2 / 3$ of the bottom of the crypt injured; $3=$ total crypt loss but epithelial lining remains; and $4=$ crypt and epithelial loss. The ulceration was graded according to the worst area. Then, the ulceration score was multiplied by the length of involved colon. The individual ulceration scores were then summed and divided by the total colon length to give a measure of ulceration severity/ unit colon. The percentage of colon scored per grade for each mouse was calculated and used to allow comparison of the spread of grades between genotypes.

\section{Immunohistochemistry}

Two different human colon tissue microarrays, both containing some specimens of human IBD, were purchased from US Biomax (Rockville, MD, USA). For mouse tissues, formalinfixed, paraffin-embedded $5 \mu \mathrm{m}$ sections were used. The slides were dewaxed, rehydrated, and treated with hydrogen peroxide to quench endogenous peroxidases. After heat-mediated antigen retrieval using $10 \mathrm{mM}$ sodium citrate, $\mathrm{pH}$ 6.0, the slides were blocked for $1 \mathrm{~h}$ with $5 \%$ goat or rabbit serum and then incubated overnight with a rabbit polyclonal anti-human MMP10 antibody (Abcam, Cambridge, MA, USA), a rat anti- 
mouse neutrophil antibody (Serotec, Raleigh, NC, USA), or a rat anti-mouse F4/80 antibody (Serotec). The following day, the slides were washed with Tris-buffered saline and incubated with biotinylated anti-rabbit or anti-rat antibodies (Vector Laboratories, Burlingame, CA, USA) before being incubated with Vectastain $\mathrm{ABC}$ reagent (Vector Laboratories) following the manufacturer's instructions. The chromogen diaminobezidine (DAB; Sigma) was used to visualize positive antibody binding, and the slides were counterstained with Mayer's hematoxylin before clearing and mounting. Quantification of positive signals from the neutrophil and macrophage immunostaining was done using Metamorph software on images taken with a $20 \times$ objective lens with a Zeiss Axiophot microscope, as previously described.

\section{Immunofluorescence}

Colon from mice treated for 7 days with $2.5 \%$ DSS was harvested, rinsed, split open, rolled, covered with OCT freezing medium, and quick frozen in liquid nitrogen. Then, $8 \mu \mathrm{m}$ sections were cut onto glass slides and rinsed in PBS before fixing in acetone for $5 \mathrm{~min}$ and then blocking with Image-iT FX signal enhance reagent (Life Technologies, Carlsbad, CA, USA) for $30 \mathrm{~min}$ followed by $10 \%$ goat serum for $15 \mathrm{~min}$. The slides were incubated for $2 \mathrm{~h}$ at room temperature with rat anti-F4/80 (Serotec) and/or rabbit anti-MMP10 (Novus Biologicals, Littleton, CO, USA) in 10\% serum. After washing, the slides were incubated with AlexaFluor488 anti-rat and AlexaFluor594 anti-rabbit antibodies (Life Technologies) for $30 \mathrm{~min}$. After repeated washing and a $2 \mathrm{~min}$ incubation in $0.5 \mu \mathrm{g} / \mathrm{ml}$ Hoechst 33258 (Sigma) diluted in PBS, the slides were mounted using Prolong Gold Anti-Fade (Life Technologies). The slides were examined with a Zeiss Axiophot microscope and $20 \times$ objective lens images captured with a Hamamatsu camera using Metamorph software.

\section{In Situ Hybridization}

A 750-bp sequence of mouse $M M P 10^{16}$ carried in the pGem4mStr2 plasmid, a gift from Dr Lynn Matrisian, Vanderbilt University, was labeled according to the manufacturer's instructions using the Dig T7/Sp6 labeling kit (Roche Applied Bioscience, Indianapolis, IN, USA) to produce sense and antisense probes. Then, $5 \mu \mathrm{m}$ paraffin sections of Swiss-rolled colons from control or DSS-exposed wild-type and $M m p 10^{-1-}$ mice were dewaxed, rehydrated and postfixed in $4 \%$ paraformaldehyde. The sections were digested with $10 \mu \mathrm{g} / \mathrm{ml}$ proteinase $\mathrm{K}$ for $20 \mathrm{~min}$, and then immersed in $0.2 \mathrm{~N}$ $\mathrm{HCl}$ for $1 \mathrm{~h}$ to quench endogenous alkaline phosphatase activity. Following $10 \mathrm{~min}$ of immersions in $0.1 \mathrm{M}$ triethanolamine and $0.1 \mathrm{M}$ triethanolamine $/ 0.25 \%$ acetic acid, to inhibit electrostatic interactions, the sections were air-dried for several hours. For hybridization, sense or antisense probes diluted to $1.5 \mathrm{ng} / \mathrm{ml}$ with hybridization buffer (Dako Cytomation, Ventura, CA, USA) were placed on individual sections in a humid chamber. Each section was covered with a hybri-slip (Sigma), and the chamber incubated at $50{ }^{\circ} \mathrm{C}$ for
$16 \mathrm{~h}$. Following hybridization, the slides were washed in three changes of $2 \times \mathrm{SSC} / 50 \%$ formamide at $50^{\circ} \mathrm{C}$, and then in $2 \times$ SSC at room temperature. The presence of probe was detected using an alkaline phosphatase-labeled anti-digoxigenin antibody (Roche) that was incubated with the sections for $2 \mathrm{~h}$, according to the manufacturer's recommendations. Following washing, colorimetric signal was developed using the substrates BCIP and NBT (Promega, Madison, WI, USA).

\section{Quantitative PCR}

Isolation and analysis was performed as previously described. ${ }^{17}$ Briefly, total RNA was isolated using Trizol (Invitrogen, Carlsbad, CA, USA) and used to generate cDNA with a High Capacity cDNA archive kit (Applied Biosystems, Foster City, CA, USA). Then, $5 \mu \mathrm{g}$ of the cDNA was mixed with primers and FAM-labeled Taqman probes for MMP10 and GAPDH, and product was measured using an ABI- HT7900 Fast Real-Time PCR system. The threshold cycle $(\mathrm{Ct})$ was obtained from duplicate samples and averaged, and the $\mathrm{Ct}$ range for each is shown in the figure legends. The $\Delta \mathrm{Ct}$ was the difference between the average $\mathrm{Ct}$ for the specific cDNAs and the average $\mathrm{Ct}$ for GAPDH, which ranged from 16.97 to 14.16 . The $\Delta \Delta \mathrm{Ct}$ was the average $\Delta \mathrm{Ct}$ at a given time point minus the average $\Delta \mathrm{Ct}$ of day 0 samples. The data are expressed as relative quantification (RQ), which is the fold change and calculated as $2^{-\Delta \Delta \mathrm{Ct}}$. For measurement of macrophage markers, specific Quantitect primers for Nos2 (INOS), Cxcl10, Ccl3, Chi3l3 (Ym1), Retnla (FIZZ), Arg1 (arginase), and Gapdh were obtained from Qiagen and transcripts were detected using SYBR Green that had been included in the QuantiFast reaction mixture (Qiagen). Analysis was performed as above.

\section{In Vivo Permeability Measurement}

An $80 \mathrm{mg} / \mathrm{ml}$ solution of FITC-dextran (4000 MW; Sigma) was prepared with sterile saline and administered to 5 each control and $\mathrm{Mmp} \mathrm{O}^{-1-}$ mice after 0 or 3 days of $2.5 \%$ DSS treatment by oral gavage at a dose of $600 \mathrm{mg} / \mathrm{kg}$. After $4 \mathrm{~h}$, serum was collected from the gavaged mice and assayed using a fluorometric plate reader (Victor3 Multi-Label Counter, Perkin-Elmer) for levels of green fluorescence from the FITCdextran.

\section{Evaluation of Cytokines and Chemokines}

Multi-array ELISA plates for both cytokines and chemokines were purchased from SA Biosciences (Frederick, MD, USA). Pooled plasma or colonic lavage fluid from 3 each control or $M m p 10^{-1-}$ mice collected at 0,4 , or 8 days after initiation of DSS exposure were used for these assays, which were performed according to the manufacturer's instructions. Only those cytokines/chemokines that showed differences between the control and $\mathrm{Mmp}_{10} \mathrm{O}^{-/-}$groups were evaluated further. Single analyte ELISAs for the cytokines interleukin (IL)-1, interferon- $\gamma$ (IFN- $\gamma$ ), G-CSF, and TNF, and the chemokine monocyte chemoattractant protein-1 (MCP-1), were 
purchased from SA Biosciences. Plasma or lavage fluid from individual mice collected at 0,4 , or 8 days after initiation of DSS exposure were analyzed following the manufacturer's recommendation.

\section{Macrophage Cell Culture}

RAW264.7 murine monocyte cells were obtained from American Tissue Culture Collection (ATCC, Rockville, MD, USA) and maintained as recommended in Dulbecco's modified Eagle's medium (DMEM; Life Technologies) containing $10 \%$ fetal calf serum. For activation in vitro, the cells were treated with $10 \mathrm{ng} / \mathrm{ml}$ lipospolysaccharide (LPS; Sigma) plus $100 \mathrm{IU} / \mathrm{ml}$ IFN- $\gamma$ (R\&D Systems, Minneapolis, MN, USA) or $10 \mathrm{ng} / \mathrm{ml} \mathrm{IL-4} \mathrm{(Life} \mathrm{Technologies)} \mathrm{plus} 10 \mathrm{ng} / \mathrm{ml}$ IL-13 (Life Technologies) for $24 \mathrm{~h}$.

\section{RESULTS \\ MMP10 mRNA Expression Is Increased in Mice Exposed to DSS}

MMP10 is expressed by ulcer edge enterocytes and cells within granulation tissue in human UC. ${ }^{8,18}$ To assess possible function of this enzyme in colitic disease, we first determined when MMP10 is expressed in a mouse model of acute colitis. Mice were exposed to $2.5 \%$ DSS provided ad libitum in drinking water for a period of 7 days, and then returned to regular drinking water. At days $0,7,10,14,21$, and 28 after initiation of DSS, groups of mice were killed and RNA prepared from the colonic tissue. As can be seen in Figure 1a, MMP10 expression was low to absent in normal colonic tissue, rose to peak expression at the end of the DSS treatment, and then gradually returned to control levels. Based on $\mathrm{Ct}$ values $>35$, we conclude that MMP10 was not expressed in normal colon but was induced in response to acute colon injury, and that expression is turned off once the tissue has repaired and inflammation has resolved. Semiquantitative reverse transcription-PCR using a different set of primers confirmed these results (data not shown).

\section{Infiltrating Cells Are Responsible for the Majority of MMP10 Seen in Murine and Human IBD}

Animals exposed to $2.5 \%$ DSS predictably developed colitic damage and associated inflammation, which was histologically confirmed by crypt loss as well as infiltration of a large number of cells. As we found antibodies to mouse MMP10 to be unreliable for immunohistochemistry in paraffin sections, we used in situ hybridization to identify the cell types responsible for the production of MMP10 in colitic areas. The strongest positive signal was seen in infiltrating cells surrounding the bottom of crypts (Figure 1b). To ascertain whether such expression was limited to the DSS acute colitis model, we also stained sections from IL-10-null mice, which develop spontaneous chronic IBD. ${ }^{19}$ As can be seen in Figure 1c, there was a similar pattern of MMP10 positivity in infiltrating cells within colonic lesions. To determine whether the pattern of expression was similar in human IBD, we examined archival de-identified human colon tissue specimens from IBD patients. Although there was some evidence of epithelial expression as reported in earlier literature, ${ }^{18}$ by far the strongest expression was in inflammatory cells (Figure 1d). Overall, 6 out of 15 sections showed MMP10 staining in epithelial cells, 13 out of 15 showed MMP10 staining in inflammatory cells, and 2 out of 15 had no detectable MMP10. To determine whether the MMP10expressing cells were macrophages, as suggested by morphology, we used a double-staining immunofluorescent procedure on frozen sections of colon tissue from mice that had been exposed to $2.5 \%$ DSS for 7 days. As shown in Figure 1e, the majority of the MMP10-positive cells in the sub-crypt area also stained for the macrophage marker F4/80.

\section{MMP10-Null Mice Have More Severe DSS Colitis}

To determine the functional significance of MMP10 in acute colitis, we placed cohorts of wild-type and $M m p 10^{-/-}$mice on DSS treatment for 7 days, and then allowed subgroups of each genotype to heal for different periods of time (Figure 2a). To measure the severity of DSS colitis, we monitored weight loss, colon length, and histologic score. Mice consistently reached the nadir of their weight loss at 8-10 days after DSS initiation (Figure 2b). At the 10-day time point, the weight loss of the $\mathrm{Mmp}_{10^{-/-}}$mice was significantly worse in the 10-, 14-, and 21-day groups. Notably, we observed no difference in either starting weight or colon lengths between control $\mathrm{Mmp} 10^{-/-}$ and wild-type mice not exposed to DSS (Supplementary Figure 1A and B). Colon shortening, a morphometric measure of inflammation was more severe in the $\mathrm{MmplO}^{-1-}$ mice (Supplementary Figure 2). Ulceration scores, generated from histological assessment, showed significant differences in the amount of severe, grade 4 ulceration in the $\mathrm{Mmp} 10^{-/-}$mice compared with wild-type controls (Figure 2c). In wild-type mice, there was almost complete resolution of histological damage to the colon by day 21 , that is, 14 days after discontinuation of the DSS. In contrast, significant tissue damage remained evident in $\mathrm{Mmp} \mathrm{O}^{-/-}$mice at this time point.

\section{Basal Proliferation and Intestinal Permeability Is Normal in $\mathrm{Mmp} 10^{-1-}$ Mice}

$M m p 10^{-1-}$ animals do not have any gross phenotypic abnormalities and reproduce normally. ${ }^{20}$ The general appearance of their colons and presence of inflammatory cells did not differ from wild-type mice (data not shown). As it has been reported that mice deficient in a related enzyme, MMP2, appear to have a defect in barrier function in the gut, ${ }^{21}$ we assessed gut permeability directly. To do this, cohorts of wild-type and $M m p 10^{-/-}$mice received a dose of FITC-dextran by oral gavage. After $4 \mathrm{~h}$, serum was collected from the gavaged mice and the levels of FITC measured using a plate fluorometer. As anticipated from the lack of baseline inflammation, permeability did not differ between control wild-type and $M m p 10^{-/-}$mice (Supplementary Figure 3A). Notably, the permeability was increased after 3 days of 

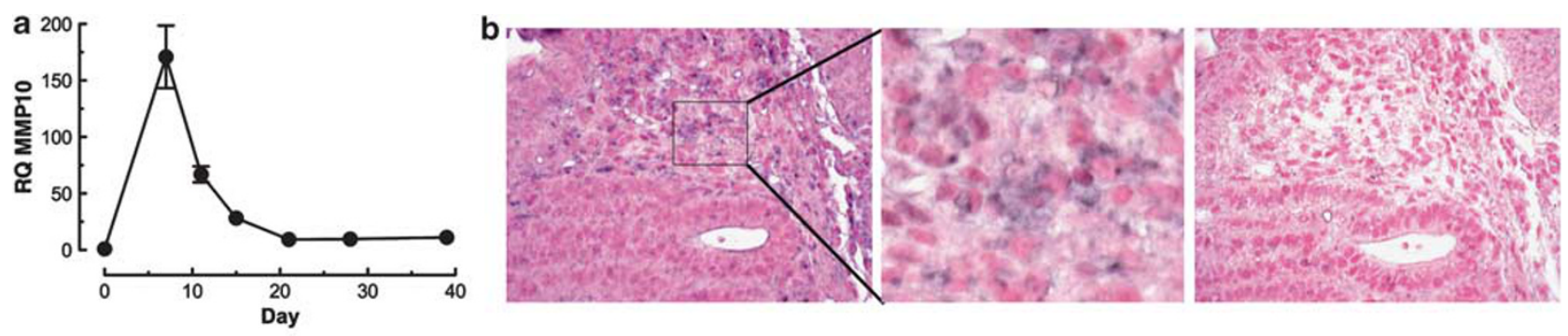

C
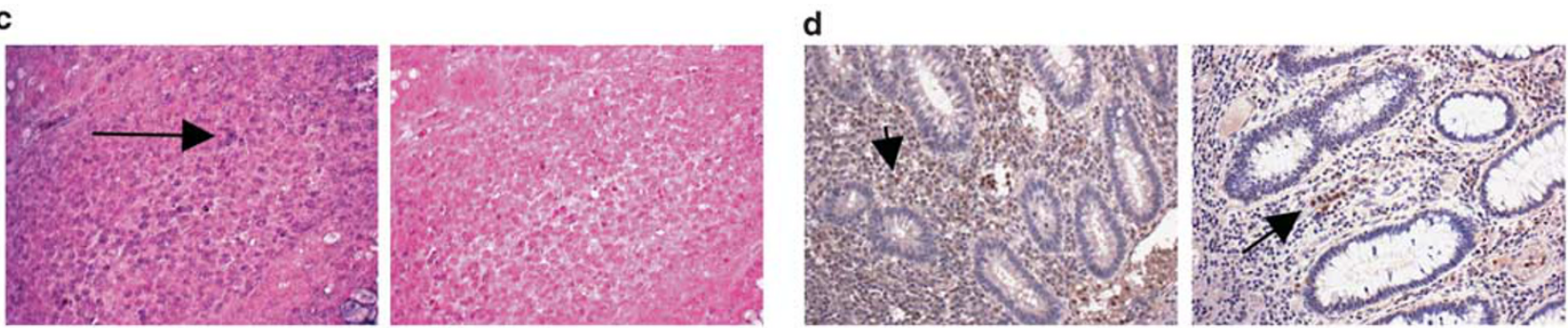

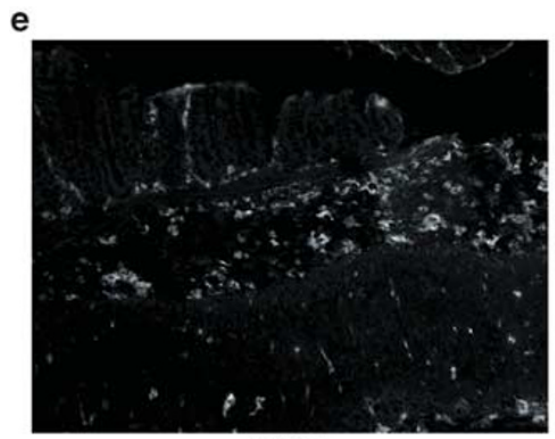

F4/80

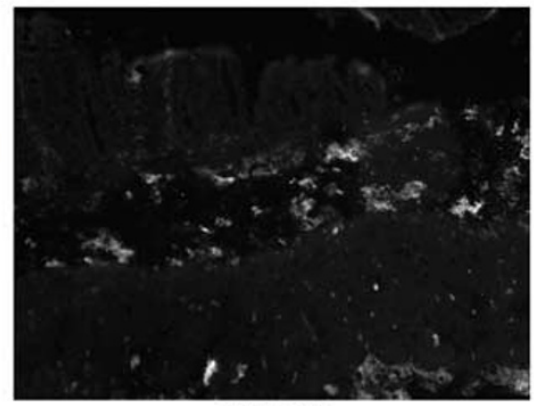

MMP10

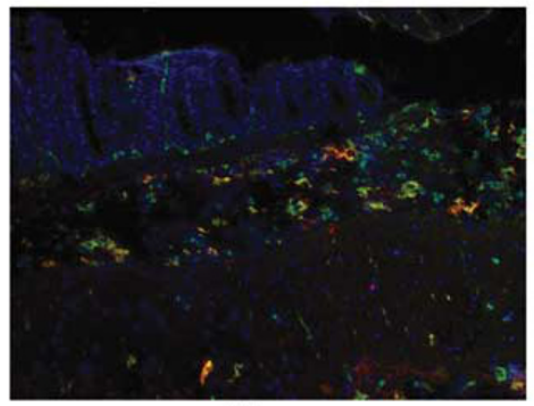

Merge

Figure 1 MMP10 is induced by inflammation in murine and human colonic tissue. (a) Real-time PCR analysis of MMP10 expression in colon tissue of C57BL/6 mice at different time points before, during, and after a 7-day treatment with DSS. (b, c) In situ hybridization for Mmp10 transcript in colon tissue specimens from DSS-treated (b) or $1110^{-1-}$ (c) C57BL/6 mice. Antisense and sense probes were both labeled with digoxigenin and applied at the same concentration. Positive signal is purple (eg, arrows); nuclei are counterstained with Fast Red. A higher magnification of the area bounded by a rectangle in the DSS antisense image is shown in the adjacent panel. (d) Immunohistochemical detection of MMP10 protein in specimens of colon tissue from two different IBD patients. Positive signal is brown; nuclei are counterstained blue with hematoxylin. (e) Immunofluorescent costaining of a frozen section of colon from a mouse after a 7-day treatment with DSS. The murine macrophage marker F4/80 is shown on the left, MMP10 is shown in the center, and merged image showing F4/80 (green), MMP10 (red), and nuclear stain (blue) is on the right.

exposure to $2.5 \%$ DSS but was still not significantly different between the two strains of mice (Supplementary Figure 3B). Immunohistochemical staining of the colonic tissue also demonstrated that untreated $M m p 10^{-/-}$and wild-type animals have no difference in the number of dividing cells identified by phosphohistone-3 immunostaining (data not shown). Thus, we concluded that the differences seen following DSS exposure were not due to baseline differences between wild-type and $M m p 10^{-/-}$mice.

Leukocyte Infiltration Was Increased in MMP10-Null Mice To better characterize the infiltrating cell populations in the colonic lesions that developed after DSS exposure, we immunostained colon sections with anti-neutrophil antibody. Although at day 7 we observed fewer neutrophils in $M m p 10^{-/-}$colons, at days 10 and 14 there was a trend for greater neutrophils in $\mathrm{Mmp} 10^{-/-}$mice compared with wild- type mice (Figure 3a). More strikingly, at day 21, when neutrophilia had largely resolved in wild-type mice, elevated numbers of neutrophils persisted in the colons on $\mathrm{Mmp} 10^{-/-}$ mice (Figure 3a). In contrast, there was a significantly higher level of macrophages at day 7 in the MMP10-null samples, coincident with the peak expression of MMP10 seen in wildtype mice (Figure 1a). Intriguingly, at day 21, although there were still large lesions visible in the $M m p 10^{-/-}$tissues, staining for $\mathrm{F} 4 / 80+$ macrophages was undetectable, similar to the situation in the healed wild-type colons. As macrophages can be activated to different functional states, and there are suggestions that alternatively activated (M2) macrophages are important in resolution of DSS-induced damage, we assessed levels of macrophage activation markers. Whole colons were isolated from 5 each wild-type and $M m p 10^{-/-}$mice that had been exposed to $2.5 \%$ DSS for 7 days, and then given 1 day to start recovery. Levels of 

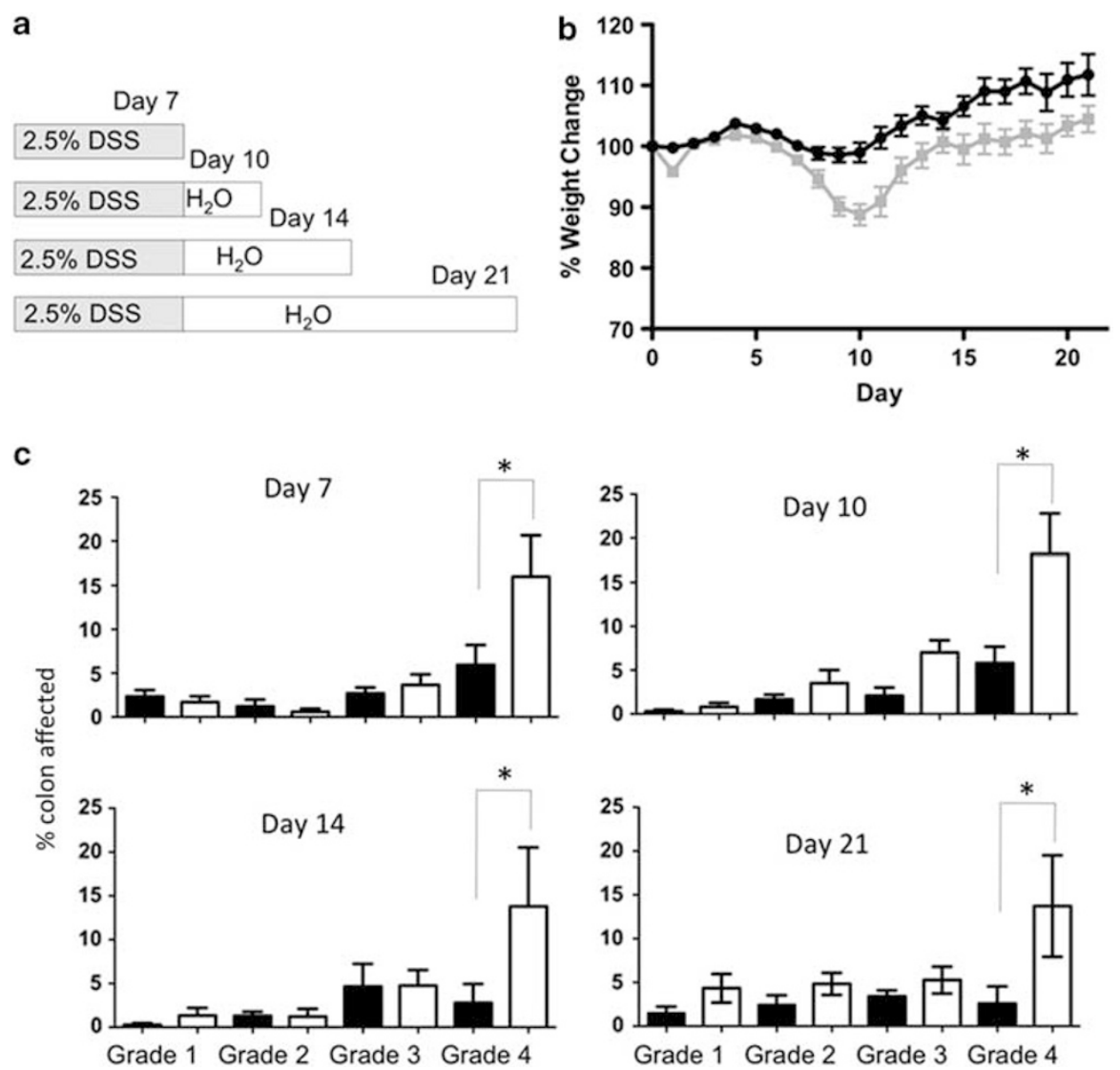

Figure 2 MMP10-deficient mice are significantly more injured by DSS treatment than wild-type mice. (a) Scheme showing the four time points after a 7-day exposure to $2.5 \%$ DSS at which cohorts of mice were analyzed. (b) Average weight change, relative to each individual starting weight, for all mice on the DSS protocol. Wild-type mice are shown in black, whereas Mmp10 - / - mice are in gray. (c) Histological scores for wild-type (black bars) and $\mathrm{MMP}^{-1} \mathrm{O}^{-1}$ (white bars) colon sections from mice assessed at the four different time points outlined in (a). The description for each of the grades is given in the Materials and Methods. Shown is the average percentage of the colon length assessed at each grade for each group of mice.

transcripts for the classically activated (M1) macrophage markers INOS, CXCL3, and CCL10, as well as for the alternative activation (M2) macrophage markers mannose receptor, Ym1, and FIZZ, were determined using real-time PCR analysis of RNA from the colon tissue. The combination of these markers for macrophage phenotyping has been described in the literature. ${ }^{22,23}$ As can be seen in Figure $3 c$, the relative levels of M1 markers were increased whereas M2 markers were decreased in the colonic tissue from DSS-treated $M m p 10^{-/-}$mice.

\section{Cytokine Profiles of Colonic Lavage Correlate with Severity of Colitis}

As MMP10 was primarily produced by inflammatory cells during DSS-induced colitis, we reasoned that MMP10 may play a role in cytokine release. To assess whether levels of secreted cytokines were different between wild-type and $M m p 10^{-1-}$ mice, colonic lavage fluid was collected from mice that had been exposed to 3-4 or 7 days of DSS. The 7-day exposure group was given 1 additional day on regular water to allow the repair process to begin before samples were collected on day 8. Samples were also collected from mice that were never exposed to DSS. Levels of cytokines were then assessed as detailed in the Materials and Methods. Levels of IL-1A, TNF- $\alpha$, and G-CSF were significantly increased in MMP10-null animals 8 days after initial exposure to DSS compared with the wild-type counterparts; however, we saw no difference in levels of any cytokine between genotypes at earlier time points (Figure $4 \mathrm{a}-\mathrm{c}$ ). In addition, the levels of other factors assayed (IL-1b, IL-2, IL-4, IL-6, IL-10, IL-12, IL-17a, interferon- $\gamma$; or GM-CSF) did not differ between wild-type and $\mathrm{Mmp} 10^{-1-}$ mice at baseline or either experimental time point (data not shown). Thus, the higher levels of specific pro-inflammatory cytokines at the late time point in the Mmp10 $10^{-/-}$mice were most likely related to a failure to resolve on-going inflammation.

As there was an apparent difference in the number of infiltrating leukocytes in the colitic lesions of DSS-treated $M m p 10^{-/-}$mice compared with controls, we also assessed levels of chemokines, both in the local environment, by testing colonic lavage, and systemically in the blood. Only one chemokine, macrophage chemoattractant protein-1 (MCP-1/ CCL2), differed between wild-type and $\mathrm{Mmp10}^{-/-}$mice, with elevated levels in null mice at both early and late stages of disease, suggesting that it may have a functional role in inducing the exacerbated inflammatory response (Figure 4d). 

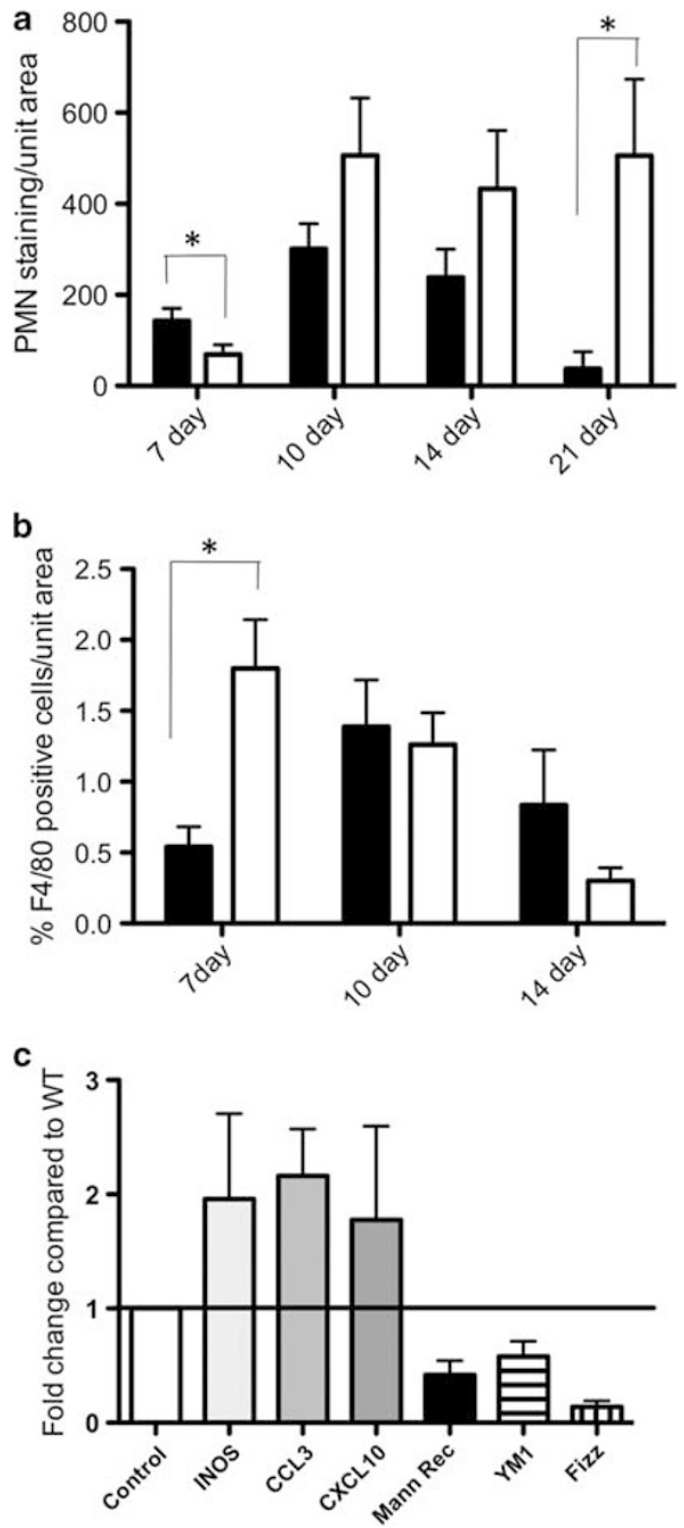

Figure 3 Leukocyte recruitment is altered in DSS-treated MMP10deficient mice. (a) The average level of PMN immunostaining per unit area of colonic tissue in wild-type (black bars) or MMP10 ${ }^{-1-}$ (white bars) mice at each of the four time points after DSS treatment. (b) The average number of F4/80-positive macrophages per unit area of colon tissue from wild-type (black bars) or MMP10 ${ }^{-1-}$ (white bars) mice at three time points after DSS treatment. Macrophages were undetectable at 21 days. (c) Whole colon lysates from 7-day DSS-treated mice were analyzed by real-time PCR for markers of macrophage activation status. Shown are the levels of markers of M1 macrophages (INOS, CXCL3, and CCL10) and M2 macrophages (mannose receptor, Fizz, and Ym1) in MMP10-null colons compared with levels in wild-type colons after normalization using levels of GAPDH.

\section{Bone Marrow Transplant Suggests Marrow Genotype Contributes to Severity of Colitis}

The identification of infiltrating cells, particularly macrophages, as the predominant cellular source of MMP10 prompted us to investigate whether the more severe colitic phenotype of the $M m p 10^{-1-}$ mice was dependent only on bone marrow cells. We lethally irradiated matched cohorts of control and $M m p 10^{-/-}$animals and repopulated with donor marrow from healthy control or $\mathrm{Mmp}^{-0^{-/-}}$mice. After a 4-week period of reconstitution, we challenged recipient mice with a 7-day course of 2.5\% DSS and then killed mice 1 day after the DSS treatment ended. Analysis of weights showed no significant differences among the groups (Figure 5a). Histological analysis, however, indicated that the extent of grade 4 severe lesions was associated predominantly with the genotype of the bone marrow supplied in $M m p 10^{-/-}$but not wild-type recipient mice (Figure 5b). It is important to note that these experiments were conducted in a specific pathogen-free barrier facility, where the severity of DSS-induced colitis was reduced in all animals compared with their counterparts in normal housing. Overall, these results suggest that bone marrow-derived MMP10 contributes to amelioration of colitis severity, but other cellular sources present in wild-type mice are also important.

\section{Incidence of Dysplasia Is Increased in $\mathrm{Mmp} \mathrm{O}^{-/-}$Mice} In order to determine whether lack of MMP10 altered colitisassociated cancer development, we attempted to induce chronic colitis by repeated exposure to DSS. Previous literature demonstrated that four rounds of DSS exposure result in chronic colitis in C57BL/6 mice. ${ }^{24}$ However, severe morbidity that reached the criteria for killing occurred in the $M m p 10^{-/-}$mice after only two rounds of DSS, barring any further exposure. Thus, we treated cohorts of wild-type and $\mathrm{Mmp} \mathrm{O}^{-/-}$mice with two 4-day rounds of $3 \%$ DSS, 3 weeks apart, and let the animals recover for a further 3 weeks before termination. We then isolated the colons and examined them histologically. As expected, the degree of dysplasia in the wild-type mice after only two rounds of DSS was very low. In $M m p 10^{-1-}$ mice, however, the frequency of dysplasias detected was significantly elevated $(6 / 8(75.0 \%)$ in Mmp10 $10^{-/-}$versus 1/7 (14.3\%) in wild type; $P=0.04, \chi^{2}$ test). Examples of the lesions detected in $\mathrm{Mmp} 10^{-/-}$mice are shown in Figure 6.

\section{DISCUSSION}

Here we have used the DSS model of acute intestinal damage and inflammation to investigate functions of the proteinase MMP10. Although MMP10 expression was induced in response to colon injury, our data indicate that this proteinase functions more in disease resolution than progression. Mice lacking MMP10 were clearly deficient in their ability to effectively heal mucosal ulcers. These results led us to characterize MMP10 as playing a beneficial/protective role in the DSS-induced colitic damage model, and suggest that strategies to enhance MMP10 rather than inhibit its activity may have therapeutic impact.

One difficulty with testing possible molecular mechanisms for IBD and associated therapeutic strategies is the paucity of models that can truly recapitulate the complexity of human 

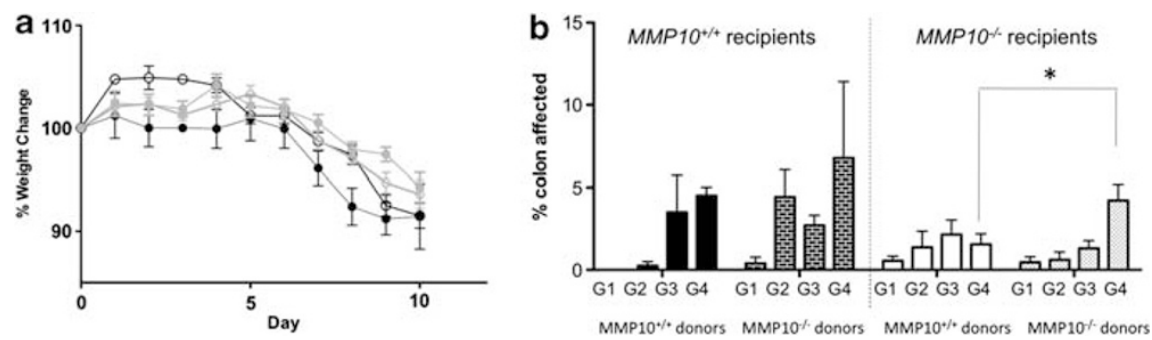

Figure 4 Bone marrow transplant experiments suggest colitis severity is largely controlled by bone marrow-derived MMP10. (a) Weight changes over the course of DSS treatment and 3-day recovery. Black symbols indicate wild-type recipients, whereas gray indicates MMP10 ${ }^{-1-}$ recipients. Filled circles indicate wild-type donors, whereas empty circles indicate $\mathrm{MMP}^{-1-}$ donors. (b) Histologic grades of colonic tissue specimens from bone marrow transplant mice. The dark bars on the left indicate wild-type recipients, whereas the light bars on the right are MMP10 ${ }^{-1-}$ recipients. Solid bars are wild-type donors and hashed bars are MMP10-1- donors. The grades (assigned as described in the Materials and Methods) are indicated by G1 through G4, with the bars representing the average percentage of colon length calculated for each grade in each group of mice.
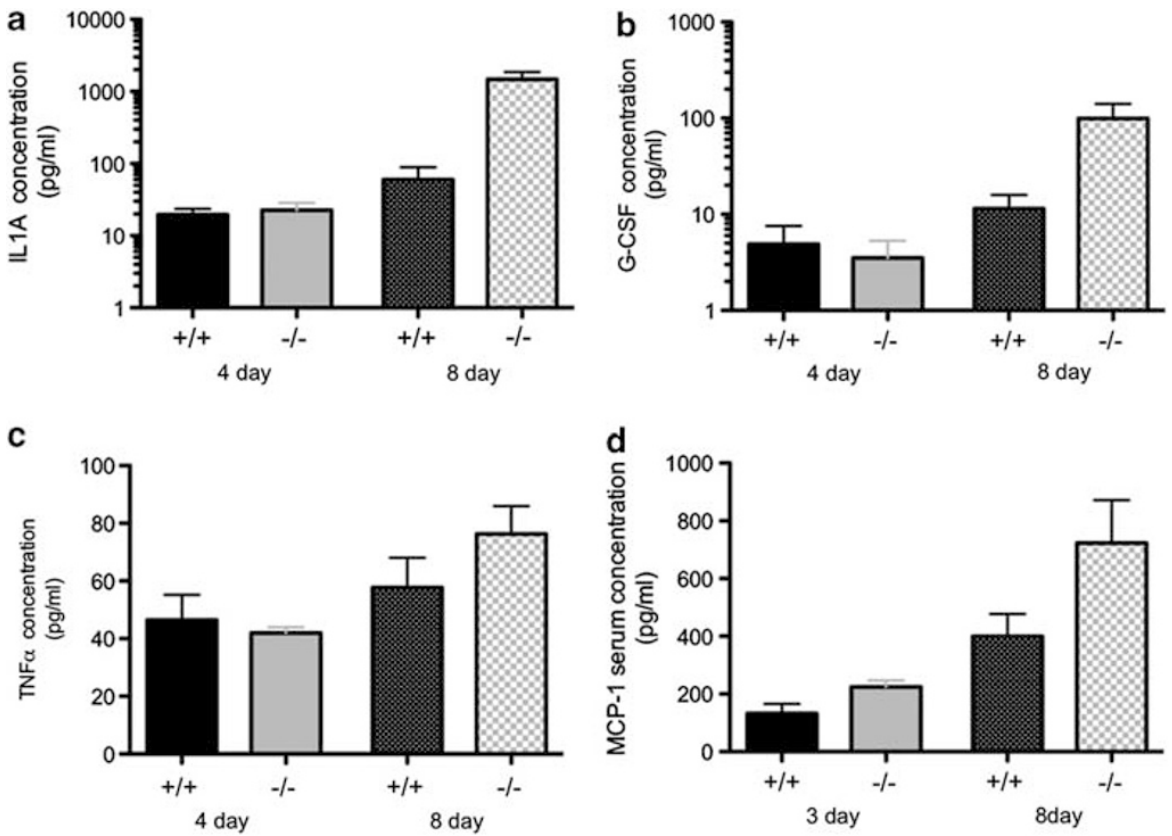

Figure 5 Levels of cytokines and chemokines detected by ELISA in fluids from DSS-treated mice. Levels of IL-1a (a), G-CSF (b), and TNF- $\alpha$ (c) measured in colonic lavage samples collected from mice on day 4 or day 8 of a 7-day DSS treatment protocol. Day 8 mice had 1 recovery day before collection. (d) Levels of the chemokine MCP-1 detected in serum from mice on day 3 or day 8 of a 7-day DSS treatment protocol.

disease. Nevertheless, particular aspects of the disease process such as immune cell dysregulation or epithelial damage can be examined using the IL-10 $10^{-/-}$mouse, ${ }^{19}$ transplantation of $\mathrm{CD} 45^{\text {hi }} \mathrm{CD} 4+\mathrm{T}$ cells into immunosuppressed animals, ${ }^{25}$ or treatment with agents such as $\mathrm{TNBS}^{26}$ or DSS, ${ }^{27}$ respectively. The DSS model, in particular, is widely used because of the ease of administration and ability for temporal control. ${ }^{27}$ The detailed mechanism by which DSS induces damages and ensuing acute inflammation has been recently described. ${ }^{28}$ One attractive feature of this model is different strains of mice and other rodents are differentially sensitive to the effects of DSS, reflecting the role that genetic factors have in human disease. ${ }^{15}$ Thus, although the DSS model cannot be considered 'mouse ulcerative colitis', aspects of it are similar and it has been useful in identifying some important pathways as well as for testing of drugs. ${ }^{27}$
A previous report of MMP10 in human IBD suggested a possible role in healing; however this was based solely on apparent localization in migrating epithelial cells. ${ }^{18}$ Our investigation of MMP10 localization in DSS-injured and IL10-null mouse colons and in human IBD samples revealed more prominent expression in leukocytes rather than epithelial cells, a pattern also seen in models of lung infection and injury (WC Parks, unpublished observations). From their morphology, the MMP10-positive cells were of a monocytic origin. In the DSS model, we then confirmed macrophage expression of MMP10 using costaining with the widely used murine macrophage marker F4/80. ${ }^{29}$ Although $\mathrm{F} 4 / 80$ is predominantly expressed by macrophages, there can be some expression by subclasses of dendritic cells and eosinophilic granulocytes; therefore, there remains a formal possibility that myeloid cells other than macrophages express MMP10 in the 

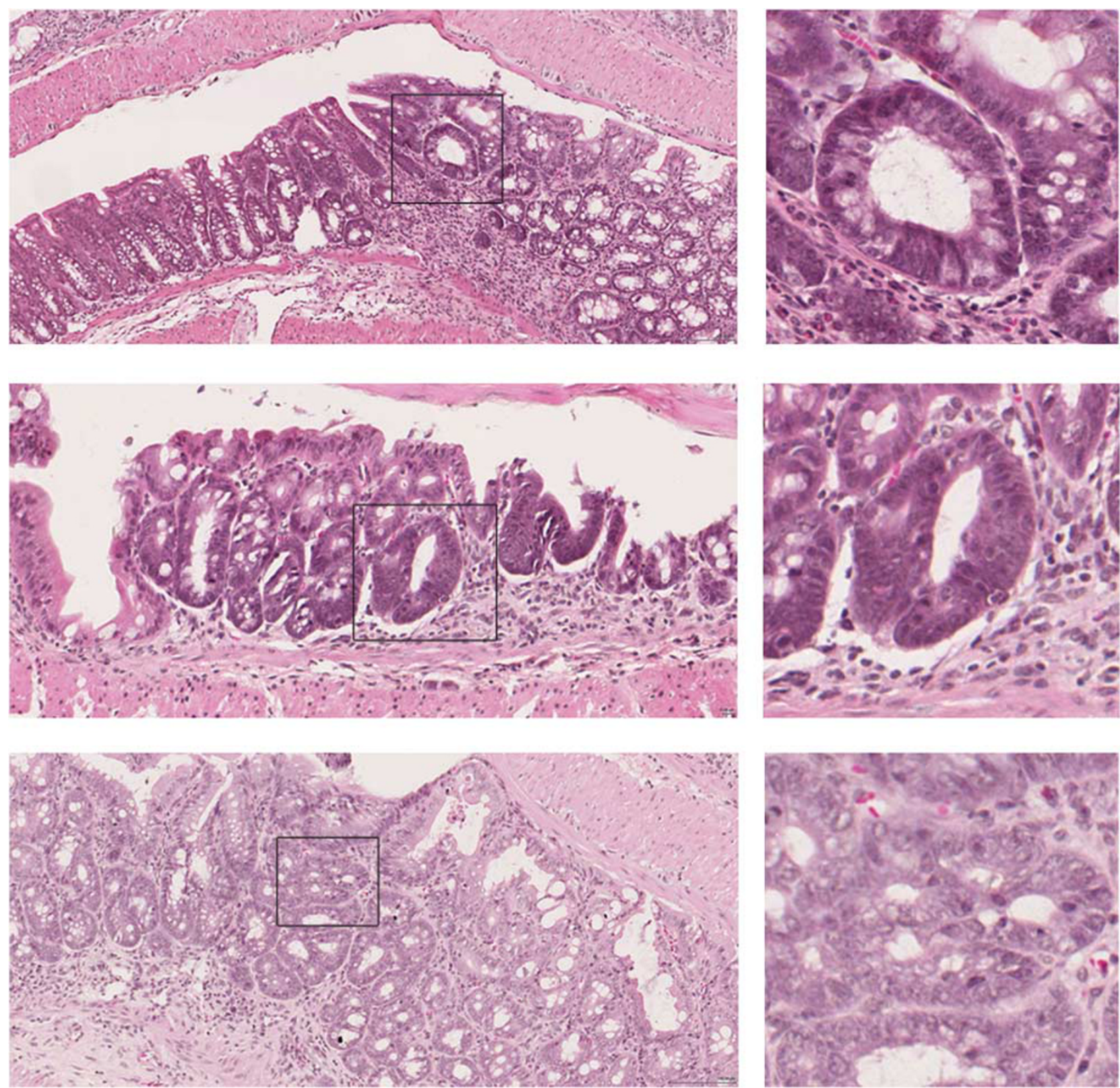

Figure 6 Dysplastic lesions are present in the colons of $\mathrm{MMP} 0^{-/-}$mice after two rounds of DSS treatment. Shown are examples of hematoxylin and eosin-stained lesions from three different $\mathrm{MMP}_{10}{ }^{-1-}$ mice. Boxed regions showing examples of dysplasic lesions are shown in higher magnification on the right. Scale bar $=100 \mu \mathrm{m}$.

DSS model. Our bone marrow transplant experiments support the premise that MMP10 in bone marrow-derived cells provides a protective response to DSS-induced injury. This is most clearly shown when the bone marrow is transplanted to $M m p 10^{-/-}$recipients. As the effect is not so clear in wildtype mice, MMP10 from non-bone marrow-derived cells must also contribute to regulation of colitis severity.

As MMPs are often thought of as extracellular matrixdegrading enzymes, it is unsurprising that they are often thought of as being destructive. Indeed, many previous studies of MMPs in several colitis models have confirmed deleterious functions. ${ }^{10,11,30-33}$ A common strategy for such studies was the administration of broad-spectrum MMP inhibitor drugs to experimental animals, and observation of disease modification. However, such an approach can obfuscate differential roles for individual family members. An alternative approach has been the use of in vivo administered silencing RNAs (siRNAs) toward individual members. Such reagents have been used to target both MMP3 and MMP10 in DSS-induced colitis; ${ }^{34}$ however, the level of knockdown achieved ( $\sim 50 \%$ ), the length of time for which knockdown is achieved, and the unknown penetration of the knockdown into different cell types means that results are very difficult to interpret. The availability of mouse lines 
genetically ablated for specific MMPs has allowed more careful probing of the contributions of particular enzymes. ${ }^{35}$ For example, the gelatinases MMP2 and MMP9 appear to have opposing roles, with MMP9 being destructive and MMP2 protective because of the effects on maintenance of intestinal barrier function. $^{21}$ Protective functions for individual family members have also been seen in other disease settings such as cancer. ${ }^{36,37}$ Indeed, the same MMP can have both protective and destructive roles that are context dependent. ${ }^{36}$ Together, these findings illustrate that the once popular therapeutic strategy of broad MMP inhibition is flawed. Our data would suggest that MMP10 inhibition is likely to enhance disease severity and increase healing time. It remains to be seen if increasing the levels of MMP10 would have the opposite effect and reduce healing time. If so, this could prove a valuable tool in minimizing the clinical problems associated with IBD.

One of the most dangerous effects of chronic IBD is the increased risk for colon cancer development. Colitis-associated colon cancer, although in many ways similar to sporadic colon cancer, does have some particular characteristics. It is associated with younger age at onset, a mucinous/ signet ring histology, a higher rate of 2 or more synchronous primary tumors, and a more proximal distribution in the colon. ${ }^{2}$ In the setting of IBD, cancers are believed to progress from no dysplasia to 'indefinite' dysplasia, to low-grade and then high-grade dysplasia, and ultimately becoming carcinoma. ${ }^{2}$ In our studies, a lack of MMP10 resulted in enhanced development of dysplasia in DSS-treated mice. Although we cannot rule out direct MMP10-mediated changes in epithelial cells, the most likely explanation is that the unremitting inflammation seen in DSS-treated $M m p 10^{-1-}$ mice is the major cause. In other mouse models of colon cancer such as $A p c^{\mathrm{Min/+}}$, as well as in human colon cancer specimens, MMP10 levels are often increased, ${ }^{38,39}$ although this is in multiple cell types including epithelial tumor cells. It is as yet unclear if the enzyme contributes to cancer progression or if its increased levels are merely an indicator of increased numbers of cancer cells. Future studies will address possible roles of MMP10 in colorectal cancer development and progression.

A major question remaining from our studies is the identification of the MMP10 substrate(s) responsible for its protective function. As persistent inflammation is the dominant phenotype associated with MMP10 loss in both this study and in a previously published Pseudomonas aeruginosa infection model, ${ }^{20}$ cleavage of a cytokine/chemokine may be responsible for regulating immune response. MMPmediated processing of chemokines has been reported in several systems, ${ }^{14}$ and other studies have shown MMPdependent effects on chemokine compartmentalization. ${ }^{40,41}$ Such modifications usually produce proteins reduced in only a small number of amino acids, and hence these changes are unlikely to be detected using ELISA methodologies such as were used here. We did find a change in the circulating levels of MCP-1 at early time points after DSS treatment that suggest that this may be an important effector of the enhanced inflammatory response in $\mathrm{Mmp} 10^{-1-}$ mice. It should be noted, however, that there are no apparent disease differences between the wild-type and $\mathrm{Mmp} \mathrm{O}^{-{ }^{-}}$mice at early time points after DSS exposure. Intestinal permeability is similarly increased between the two genotypes compared with their baseline counterparts (Supplementary Figure 3B), and a pilot study assessing histological changes also showed no quantifiable difference between the genotypes after 4 days of DSS exposure (data not shown).

Although increased inflammation was evident in the DSStreated $\mathrm{Mmp} \mathrm{O}^{-/-}$mice, and neutrophil numbers were particularly high, the numbers of F4/80-positive macrophages decreased over time in DSS-treated $\mathrm{Mmp} 10^{-/-}$mice compared with the corresponding wild-type mice. Interestingly, the phenotype of the DSS-treated $M m p 10^{-1-}$ mice is strikingly similar to one reported by Qualls et $a l,{ }^{42}$ in which macrophages were either systemically or locally depleted. More recently, CX3CR1-deficient mice were shown to have significant reduction in lamina propria macrophages and have enhanced severity of DSS-induced colitis. ${ }^{43}$ Other studies have suggested that macrophages are required for wound healing in models of colonic damage. ${ }^{44,45}$ Finally, there are indications that alternatively activated or M2 macrophages can be protective against colitis induced by either TNBS $^{46}$ or DSS. ${ }^{47}$ In our study, not only are macrophage numbers lower in $M m p 10^{-1-}$ mice, but there appears to be a skewing toward M1 rather than M2 macrophages. Overall then, the changes in macrophages seen in DSS-treated $\mathrm{Mmp} 10^{-/-}$mice are likely contributing factors to the failure of the healing program in these mice.

In conclusion, the data presented here indicate that MMP10, produced by infiltrating myeloid cells after DSS-induced colonic damage, plays a role in disease resolution. In the absence of this enzyme, colonic inflammation persists and eventually results in the development of dysplastic lesions. Thus, enhancing MMP10 expression may be a therapeutic strategy worth considering for promotion of mucosal healing.

Supplementary Information accompanies the paper on the Laboratory Investigation website (http://www.laboratoryinvestigation.org)

\section{ACKNOWLEDGEMENTS}

We are especially thankful to Dr Mark Frey of the University of Southern California for paraffin sections from IL-10-null mice, to Dr Lynn Matrisian for the MMP10 in situ hybridization probe, and to the Vanderbilt Tissue Acquisition Core for the archival de-identified human IBD specimens. Additionally, we gratefully acknowledge Dr MK Washington for helpful discussions regarding histology scoring, and Dr JT Roland and the Epithelial Biology Center Shared Imaging Resource for Ariol scanning of slides. FLK was supported by training Grant T32CA106183. This work was also supported by the Vanderbilt-Ingram Cancer Center support Grant P30CA068485, by pilot funding from the Vanderbilt Digestive Disease Research Center P30DK058404, and by HL089455 and HL098067 (WCP). 


\section{DISCLOSURE/CONFLICT OF INTEREST}

The authors declare no conflict of interest.

1. Osterman MT, Lichtenstein GR. Chapter 112-ulcerative colitis. In: Feldman M, Friedman LS, Brandt LJ (eds) Sleisenger's and Fordtran's Gastrointestinal and Liver Disease. 9th edn. (, Philadelphia, PA, 2010) pp1982.

2. Itzkowitz SH, Yio X. Inflammation and cancer IV. Colorectal cancer in inflammatory bowel disease: the role of inflammation. Am J Physiol Gastrointest Liver Physiol 2004;287:G7-17.

3. Sandborn WJ, Loftus EV. Balancing the risks and benefits of infliximab in the treatment of inflammatory bowel disease. Gut 2004;53:780-782.

4. $\mathrm{Ng} \mathrm{SC}, \mathrm{Kamm}$ MA. Therapeutic strategies for the management of ulcerative colitis. Inflamm Bowel Dis 2009;15:935-950.

5. Baugh MD, Perry MJ, Hollander AP, et al. Matrix metalloproteinase levels are elevated in inflammatory bowel disease. Gastroenterology 1999;117:814-822.

6. von Lampe B, Barthel B, Coupland SE, et al. Differential expression of matrix metalloproteinases and their tissue inhibitors in colon mucosa of patients with inflammatory bowel disease. Gut 2000;47:63-73.

7. Matsuno $\mathrm{K}$, Adachi $\mathrm{Y}$, Yamamoto $\mathrm{H}$, et al. The expression of matrix metalloproteinase matrilysin indicates the degree of inflammation in ulcerative colitis. J Gastroenterol 2003;38:348-354.

8. Vaalamo $M$, Karjalainen-Lindsberg ML, Puolakkainen $\mathrm{P}$, et al. Distinct expression profiles of stromelysin-2 (MMP-10), collagenase-3 (MMP13), macrophage metalloelastase (MMP-12), and tissue inhibitor of metalloproteinases-3 (TIMP-3) in intestinal ulcerations. Am J Pathol 1998;152:1005-1014.

9. Saarialho-Kere UK, Vaalamo M, Puolakkainen $\mathrm{P}$, et al. Enhanced expression of matrilysin, collagenase, and stromelysin-1 in gastrointestinal ulcers. Am J Pathol 1996;148:519-526.

10. Di Sebastiano $P$, di Mola FF, Artese $L$, et al. Beneficial effects of Batimastat (BB-94), a matrix metalloproteinase inhibitor, in rat experimental colitis. Digestion 2001;63:234-239.

11. Sykes AP, Bhogal R, Brampton $C$, et al. The effect of an inhibitor of matrix metalloproteinases on colonic inflammation in a trinitrobenzenesulphonic acid rat model of inflammatory bowel disease. Aliment Pharmacol Ther 1999;13:1535-1542.

12. Coussens LM, Fingleton B, Matrisian LM. Matrix metalloproteinase inhibitors and cancer: trials and tribulations. Science 2002;295: 2387-2392.

13. Egeblad M, Werb Z. New functions for the matrix metalloproteinases in cancer progression. Nat Rev Cancer 2002;2:161-174.

14. Parks WC, Wilson CL, Lopez-Boado YS. Matrix metalloproteinases as modulators of inflammation and innate immunity. Nat Rev Immunol 2004;4:617-629.

15. Mähler M, Bristol IJ, Leiter EH, et al. Differential susceptibility of inbred mouse strains to dextran sulfate sodium-induced colitis. Am J Physiol 1998:274:G544-G551.

16. Gack S, Vallon R, Schmidt J, et al. Expression of interstitial collagenase during skeletal development of the mouse is restricted to osteoblastlike cells and hypertrophic chondrocytes. Cell Growth Differ 1995;6: 759-767.

17. Swee $\mathrm{M}$, Wilson $\mathrm{CL}$, Wang $\mathrm{Y}$, et al. Matrix metalloproteinase-7 (matrilysin) controls neutrophil egress by generating chemokine gradients. J Leukoc Biol 2008;83:1404-1412.

18. Salmela MT, Pender SL, Karjalainen-Lindsberg ML, et al. Collagenase-1 (MMP-1), matrilysin-1 (MMP-7), and stromelysin-2 (MMP-10) are expressed by migrating enterocytes during intestinal wound healing. Scand J Gastroenterol 2004;39:1095-1104.

19. Kuhn R, Lohler J, Rennick D, et al. Interleukin-10-deficient mice develop chronic enterocolitis. Cell 1993;75:263-274.

20. Kassim SY, Gharib SA, Mecham BH, et al. Individual matrix metalloproteinases control distinct transcriptional responses in airway epithelial cells infected with Pseudomonas aeruginosa. Infect Immun 2007;75:5640-5650.

21. Garg P, Rojas M, Ravi A, et al. Selective ablation of matrix metalloproteinase-2 exacerbates experimental colitis: contrasting role of gelatinases in the pathogenesis of colitis. J Immunol 2006;177:4103-4112.
22. Wang $Y$, Wang YP, Zheng G, et al. Ex vivo programmed macrophages ameliorate experimental chronic inflammatory renal disease. Kidney Int 2007;72:290-299.

23. Zaynagetdinov R, Sherrill TP, Polosukhin VV, et al. A critical role for macrophages in promotion of urethane-induced lung carcinogenesis. J Immunol 2011;187:5703-5711.

24. Clapper ML, Cooper HS, Chang WC. Dextran sulfate sodium-induced colitis-associated neoplasia: a promising model for the development of chemopreventive interventions. Acta Pharmacol Sin 2007;28:1450-1459.

25. Kim HS, Berstad A. Experimental colitis in animal models. Scand J Gastroenterol 1992;27:529-537.

26. te Velde $A A$, Verstege Ml, Hommes DW. Critical appraisal of the current practice in murine TNBS-induced colitis. Inflamm Bowel Dis 2006;12:995-999.

27. Perse M, Cerar A. Dextran sodium sulphate colitis mouse model: traps and tricks. J Biomed Biotech 2012;2012:718617.

28. Laroui $\mathrm{H}$, Ingersoll SA, Liu HC, et al. Dextran sodium sulfate (DSS) induces colitis in mice by forming nano-lipocomplexes with mediumchain-length fatty acids in the colon. PLoS One 2012;7:e32084.

29. van den Berg TK, Kraal G. A function for the macrophage F4/80 molecule in tolerance induction. Trends Immunol 2005;26:506-509.

30. Medina C, Santana A, Llopis M, et al. Induction of colonic transmural inflammation by Bacteroides fragilis: implication of matrix metalloproteinases. Inflamm Bowel Dis 2005;11:99-105.

31. Medina C, Santana A, Paz MC, et al. Matrix metalloproteinase-9 modulates intestinal injury in rats with transmural colitis. J Leukoc Biol 2006;79:954-962.

32. Medina C, Santana A, Paz-Cabrera MC, et al. Increased activity and expression of gelatinases in ischemic colitis. Dig Dis Sci 2006;51:2393-2399.

33. Naito Y, Yoshikawa T. Role of matrix metalloproteinases in inflammatory bowel disease. Mol Aspects Med 2005;26:379-390.

34. Kobayashi K, Arimura Y, Goto A, et al. Therapeutic implications of the specific inhibition of causative matrix metalloproteinases in experimental colitis induced by dextran sulphate sodium. J Pathol 2006;209: 376-383.

35. Gill SE, Kassim SY, Birkland TP, et al. Mouse models of MMP and TIMP function. Methods Mol Biol 2010;622:31-52.

36. Overall CM, Kleifeld O. Tumour microenvironment - opinion: validating matrix metalloproteinases as drug targets and anti-targets for cancer therapy. Nat Rev Cancer 2006;6:227-239.

37. Martin MD, Matrisian LM. The other side of MMPs: protective roles in tumor progression. Cancer Metastasis Rev 2007;26:717-724.

38. Sinnamon MJ, Carter KJ, Sims LP, et al. A protective role of mast cells in intestinal tumorigenesis. Carcinogenesis 2008;29:880-886.

39. Sabates-Bellver J, Van der Flier LG, de Palo M, et al. Transcriptome profile of human colorectal adenomas. Mol Cancer Res 2007;5:1263-1275.

40. Li O, Park PW, Wilson CL, et al. Matrilysin shedding of syndecan-1 regulates chemokine mobilization and transepithelial efflux of neutrophils in acute lung injury. Cell 2002;111:635-646.

41. Corry DB, Rishi K, Kanellis J, et al. Decreased allergic lung inflammatory cell egression and increased susceptibility to asphyxiation in MMP2deficiency. Nat Immunol 2002;3:347-353.

42. Qualls JE, Kaplan AM, van Rooijen N, et al. Suppression of experimental colitis by intestinal mononuclear phagocytes. J Leukoc Biol 2006;80:802-815.

43. Medina-Contreras O, Geem D, Laur O, et al. CX3CR1 regulates intestinal macrophage homeostasis, bacterial translocation, and colitogenic Th17 responses in mice. J Clin Invest 2011;121:4787-4795.

44. Pull SL, Doherty JM, Mills JC, et al. Activated macrophages are an adaptive element of the colonic epithelial progenitor niche necessary for regenerative responses to injury. Proc Natl Acad Sci USA 2005; 102:99-104.

45. Seno $\mathrm{H}$, Miyoshi $\mathrm{H}$, Brown $\mathrm{SL}$, et al. Efficient colonic mucosal wound repair requires Trem2 signaling. Proc Natl Acad Sci USA 2009;106:256-261.

46. Rizzo A, Monteleone I, Fina D, et al. Inhibition of colitis by IL-25 associates with induction of alternatively activated macrophages. Inflamm Bowel Dis 2012;18:449-459.

47. Weisser SB, Brugger HK, Voglmaier NS, et al. SHIP-deficient, alternatively activated macrophages protect mice during DSSinduced colitis. J Leukoc Biol 2011;90:483-492. 